\title{
Identification of Design Considerations for Small Satellite Remote Sensing Systems in Low Earth Orbit
}

\author{
Juan Sebastián Triana ${ }^{1}$, Sebastian Bautistaํㅜ, Freddy Alexander Díaz González
}

\begin{abstract}
The Sergio Arboleda University is preparing its next satellite mission, named Libertad 2. It will carry out a system of image acquisition as a working tool for researches based on the obtained data. Complete methodologies are often used in the development of satellite missions for planning, execution and deployment, for example, the standards of the European Cooperation for Space Standardization; these methodologies, however, do not include technical specifications or requirements for the development of nano-satellites nor for their subsystems. For this reason, this article focuses on the identification of the characteristics, requirements and restrictions, which must be considered in the design of a remote sensing system for satellites under the CubeSat standard, in order to serve as a starting point for the development of the main payload of the Libertad 2 mission.
\end{abstract}

KEYWORDS: Remote sensing, PCB, Embedded systems, CMOS APS, Low earth orbit, CubeSat.

\section{INTRODUCTION}

The CubeSat satellites have won recognition as possible platforms for future scientific missions (Woellert et al., 2011; Saundau, 2008). Previous studies have determined the capabilities of these satellites and their functionality in the field of Earth observation. Daniel Selva, from Massachusetts Institute of Technology (MIT), conducted a feasibility study of remote sensing (RS) system technologies on-board small satellites, where he concluded which are the types of support technologies that can be implemented in satellite systems under the restrictions contained in the CubeSat standard. Additionally, he proposed technologies to perform spectral estimations of the state of vegetation and its biomass, particularly to determine the normalized differential vegetation index (NDVI) (Greenland and Clark, 2010).

Taking into account studies such as Selva’s research, Sergio Arboleda University is carrying out its second satellite mission, called Libertad 2, which consists of the development and deployment of a nano-satellite of 3 units designed under the standard of CubeSat. This mission aims wto validate the development of a prototype of RS system, which will open the field for academic research in Colombia, based on images that it could get; these investigations can focus on the analysis of use of the land for agriculture, water resources, planning of urban growth, among others. This article focuses on the identification of the characteristics, requirements and restrictions, which must be considered in the design of an RS system for CubeSat satellites, in order to serve as a starting point for the development of the main payload of the Libertad 2 mission. 
The main goal of this study was the identification of constraints and considerations for the technical design of RS systems with operation in Low Earth Orbits (LEO). In order to provide a starting point, the paper includes a brief account of CubeSat missions previously carried out, which have contained payload for Earth observation and whose developers have available information of their camera model and/or their development process. Then, the considerations at the level of hardware and software, which must be considered in the development of a CubeSat satellite system, will be established, emphasizing the characteristics of an Earth observation system. As an example, the development of the first prototype of RS system for the Libertad 2 mission is included, applying the specifications previously identified. Finally, there are the results' analysis, conclusions and future work.

\section{CUBESATS AS REMOTE SENSING SYSTEMS}

Several prototypes of the visible spectrum RS systems have been designed and tested in academic CubeSat missions. It was found that 176 CubeSats have been launched until 2013; 68 of them have been deployed with RS systems, of which only 8 met information from their development process. The first of these was the CubeSat XI-IV, developed by the University of Tokyo to validate the effective use of commercial of the shelf (COTS) components in the development of RS payloads (Funase et al., 2006; Enokuchi et al., 2015); this was based on the commercial module C3188A of Quasar Electronics that employs an OV7620 sensor. In the same way, the picosatellite ITU-pSAT- 1 and the Compass- 1 employed this same integrated camera module (Khurshid et al., 2013; Scholz et al., 2010). However, none of the three missions has published detailed information on the process of acquisition and storage of RS's images, for subsequent download to the ground station.

The AAU-CubeSat uses a KAC1310 manufacturer Kodak sensor as payload; this sensor makes use of direct memory access (DMA) module driver, which allows reaching high speed flow of data between the sensor and the main memory. Images were stored in 5 RAM memories of $512 \mathrm{~KB}$ and incorporated hamming-based algorithms of error detection and correction code (Alminde et al., 2003). However, they do not specify with which hardware's features the system was developed. CANX-1 satellite employed 2 complementary metal-oxide-semiconductor
(CMOS) sensors, a multispectral one (Hotel-2020) and a monochrome (ADCS-2120), but they do not give specifications of the developed control systems or the mode in which the images were transmitted (Stras et al., 2003).

The ESTCube-1 employs a CMOS MT9V011 along with a microcontroller STM32F217ZG of ARM Cortex M3 architecture that implements a dedicated interface for image sensors and additionally has a flexible static memory controller (FSMC) which allows easy connection to an external static random access memory (SRAM) (Kuuste, 2012). On the other hand, the University of Tartu does not provide information about the control software.

Despite the information provided by the developers of CubeSats with RS systems, no document was found that recorded the process of development of the actions and the considerations to be taken into account for the design, manufacture and assembly.

\section{CONSIDERATIONS FOR THE DESIGN OF REMOTE SENSING SYSTEMS OF DEPLOYMENT IN THE LEO}

The total system is designed based on a bus architecture, in which there is only one master subsystem called C\&DH and the rest of the subsystems are slaves, including the RS system, as show in Fig. 1. All the satellite subsystems are defined in the next paragraphs.

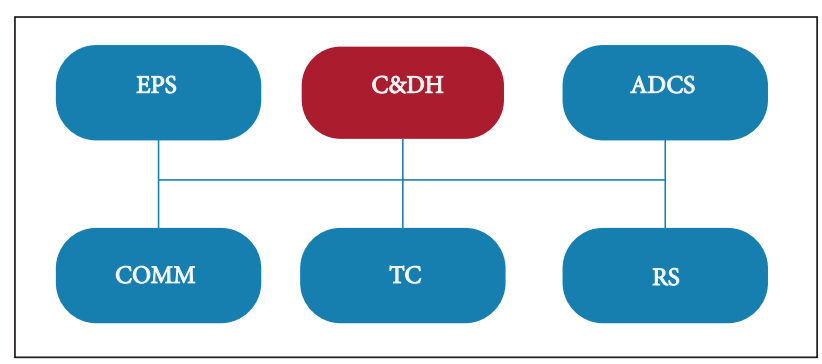

Figure 1. CubeSat subsystem's architecture (Schor et al., 2009).

To meet the goals of a satelital mission, on certain occasions, it is necessary to control the orientation of the ship, in order to perform a specific task like provide effective communication with the ground station, capture images, load the batteries with solar panels or any action required by the mission that involves movement of the satellite. All of this is possible with the attitude determination and control system (ADCS) for CubeSat.

Another fundamental factor for the fulfillment of the objectives of the mission, decisive for its lifetime, is the energy. 
Due to this, electrical power system (EPS) has autonomy in terms of energy management of the satellite; the C\&DH is able to acquire information related to the battery condition, review of the voltages, and consultation of the battery conditions.

The communication subsystem (COMM) allows the satellite be capable to communicate with Earth. For this, the C\&DH organizes the ship information into data packets to deliver them to the radio. In the same way, this subsystem receives the commands, which are related to the operations of the satellite, specific telemetry application and application data from the payload of the mission.

In the case of the RS subsystem, this would be controlled by $\mathrm{C} \& \mathrm{DH}$, which can activate the RS system at the right time to execute the programmed actions.

In the thermal control (TC), the function of the $\mathrm{C} \& \mathrm{DH}$ will focus on the reading of sensors that indicate the temperature at certain nodes of the satellite, usually obtaining data of subsystems or modules in which sudden changes in temperature may result in permanent damage to physical level or at the level of data integrity.

RS systems serve as data acquisition instruments for experiments in different fields, either of basic science or advanced research, from studies of the Earth's surface or of the deep space. These RS systems are not limited to capturing images in the visible spectrum, but they also allow to capture representations in other bands of the electromagnetic spectrum as near infrared (NIR) or infrared (IR) (Rønning, 2012). The RS system's structure (Fig. 2) can be separated into the following subsystems: a lens that focus on the incoming

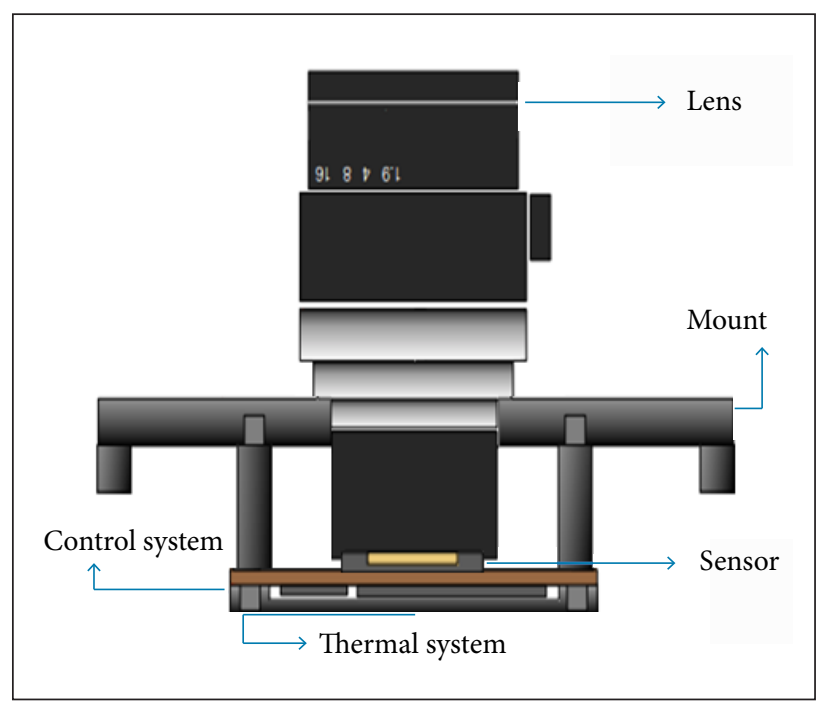

Figure 2. Image capture system structure (GomSpace, 2001). light to the sensor, which could include light filters that only allow the passing of desired frequency bands according to the study; a mount as a mechanical support that holds the lens and electronic systems in place; an image sensor that allows the system to scan the scene; a control system that manages the sensor information and a thermal system that regulates the heat on the structure given to direct exposure to sunlight due to its satellite environment; this system can be passive as a heat sink, or active as it is the case of the cooled infrared camera refrigeration systems.

\section{OPERATING ENVIRONMENT IN LOW EARTH ORBITS AND ITS IMPLICATIONS ON THE HARDWARE OF A CUBESAT}

The operation environment of these satellite systems limits the actions of support and maintenance that can be performed on it, which is why their development process must ensure robustness and fault tolerance, characteristic of a critical system (Knight, 2002). In addition, satellites in LEO, located at approximately $700 \mathrm{~km}$, turn a full Earth in approximately 95 minutes (Montenbruck et al., 2005), which means that the point on Earth to which the satellite is pointed will move with respect to the objective at $7 \mathrm{~km}$ per second; the pointing process is governed by the ADCS that guarantees the RS system pointing target for a short time period. Both the pointing process and the satellite speed leave a very precise time window in which the system is pointing to the target and the RS system is in a position ready to capture the image of the desired area. This is why the process of shooting must be synchronized with the sighting moment of the satellite to the objective, i.e. to be monitored and to ensure the exact time of execution of the image's capture process, which means it has to behave as a real time system to ensure deterministic times.

Also the restrictions surrounding a CubeSat satellite must be considered, such as the limited volume and mass and energy resources, which is why the implementation of the RS system of Libertad 2 satellite must be done on an embedded system and its software should optimize the available machine resources, including high-reliability quality attributes.

For the design of high-reliability systems for space applications, it is necessary to identify the factors that affect their behavior, in order to anticipate the failures that put at risk the goal of the mission; then, there are the most determining factors which were identified and which will be taken into account in the design of the RS system of the Libertad 2 nanosatellite. 


\section{Space Radiation in Low Earth Orbits}

Space radiation is composed of different particles such as protons, ions, electrons, among others. It comes from sources as galactic cosmic rays or solar flares. The radiation mainly induces two types of effects: the total ionizing dose (TID) and the single event effect (SEE), which affect the correct performance of the electronic devices. The TID is a cumulative energy in the integrate circuits (IC) that changes parameters like leakage current or threshold voltage, causing permanent damages. The SEE refers to particular events and occurs when highly charged particles hit the circuit. There are two types of SEE errors: the soft ones - like a single event upset (SEU), that can be corrected by software - and the hard ones, like single event latchup (SEL) and single event bur (SEB), that cause definite changes in the way the device works or even destroy it (LaBel et al., 1996).

The satellite systems in the LEO are a natural shielding by the magnetic field of the magnetosphere; however, they will be exposed to a radiation dose of about $100 \mathrm{rad} /$ year (Petkov, 2003). One way to obtain additional shielding is adding an aluminum coat of $1.5 \mathrm{~mm}$ that might prevent permanent errors in the devices (Burlyaev and van Leuken, 2014). Additionally, SEL and SEU's effects can be prevented by software through the use of Cyclic Redundancy Check (CRC) algorithms in subsystems communication, as well as through storing backup copies of each subsystem software in the master system and the use of checksum fields in stored data.

\section{Low Earth Orbits' thermal stress}

Satellites in LEO must support extreme thermal cycles due to their intermittent exposure to direct sunlight. These thermal cycles cause the outer satellite structure's temperature to oscillate from $-150^{\circ} \mathrm{C}$ to $150^{\circ} \mathrm{C}$ at its orbital period (Tennyson, 1995). In order to minimize effects on the hardware due to the thermal stress, usually a thermal control system is implemented. It regulates the heat throughout the structure in order to not let the peaks affect the components. Thermal systems can be active (heaters and coolers) or passive (thermal and insulating coatings). However, in the case of CubeSat satellite systems, an active thermal control is impractical, because the energy to maintain a stable temperature range within the structure may exceed the energy budget of the aircraft (Selva and Krejci, 2012). Due to this, the electronic components of the satellite must have a high thermal tolerance, to reduce the possibility of damage due to radiation.
For thermal management of the printed circuit board (PCB) parts, the thermal resistance must be calculated, which is the ability of a material to resist the heat flow. In the case of electronic devices operating in vacuum, it is necessary to contemplate the fact that there is not thermal resistance with the air, so there is only thermal resistance between the PCB and each device (Seo et al., 2012).

An example of calculating the thermal resistance is presented using the ADP124 (8-lead MSOP) voltage regulator, whose tolerance to external temperature should not exceed $125^{\circ} \mathrm{C}$. Equation 1 shows the thermal junction between the PCB and the device:

$T_{J}=T_{B}+\left(P_{D} \cdot \Theta_{J B}\right)$

with

$P_{D}=\left[V_{I N}-V_{O U T}\right] I_{L O A D}$

where $T_{B}$ is the PCB's temperature and $P_{D}$ is the power loss. Assuming the maximum load current $\left(I_{L O A D}\right)$ as $0.75 \mathrm{~A}$; $\mathrm{V}_{\mathrm{IN}}=3.3 \mathrm{~V}$; and $\mathrm{V}_{\text {OUT }}=1.8$ to $P_{D}$ would be:

$P_{D}=[3.3-1.8] 0.75=1.125 \mathrm{~W}$

Table 1 shows the thermal resistance coefficients between the device and the PCB according to the size of the copper zone assigned to the device die. It can be seen that the greater the area, the smaller will be the junction temperature for a given power loss. Supposing the case in which the PCB temperature reaches $75^{\circ} \mathrm{C}$ with a power loss of $1.125 \mathrm{~W}$, consider the device ADP124 should not exceed $125^{\circ} \mathrm{C}$ to operate reliably. Replacing these values into Eq. 1:

$T_{J}=T_{B}+\left(P_{D} \cdot \Theta_{J B}\right)$

$125^{\circ} \mathrm{C} \geq 75^{\circ} \mathrm{C}+\left(1.125 \mathrm{~W} \cdot \Theta_{I B}\right)$

Isolating $\Theta_{J B}$ :

$44.44^{\circ} \mathrm{C} \geq \Theta_{J B}$

$\mathrm{W}$

The operation of the ADP124 within the parameters of tolerated temperature can be guaranteed with $\Theta_{I B}=44.44^{\circ} \mathrm{C} / \mathrm{W}$ or 
Table 1. Thermal junction versus copper size.

\begin{tabular}{|c|c|}
\hline $\boldsymbol{\theta}\left({ }^{\circ} \mathbf{C} / \mathbf{W}\right)$ & Copper size $\left(\mathrm{mm}^{2}\right)$ \\
\hline 108.6 & 25 \\
\hline 75.5 & 100 \\
42.5 & 500 \\
\hline 34.7 & 1,000 \\
\hline 26.1 & 6,400 \\
\hline
\end{tabular}

less, which means that the copper prints must be at least with a size of $500 \mathrm{~mm}^{2}$. Additionally due to the asymmetry between the surfaces, it is recommended to fill the joints between the copper and the device with thermal grease and to apply thermal pads, to reduce the thermal contact resistance and the gaps as well.

\section{Low Pressures in Low Earth Orbits}

In the LEO orbit, internally, the satellites have a close to absolute vacuum pressure (Grossman and Gouzman, 2003). Some materials at low pressures tend to have effects of degassing, where particles of the material are released in the form of gas and may end up condensing optics and solar cells, affecting the proper functioning of the satellite's system. In order to mitigate the effect of degassing, materials must comply with the requirements of the ECSS-Q-ST-70-02-C standard or the ASTM E595 standard, laying down the evidence these materials must submit and their standards of acceptance for deployment in vacuum (Fayazbakhsh and Abedian, 2010).

\section{DESIGN FOR HIGH RELIABILITY OF ELECTRONIC SUBSYSTEMS FOR AEROSPACE APPLICATION}

It is common to use redundant hardware for high-reliability systems, but it is not viable for CubeSat pace applications due to the increase in energy consumption and volume limitations. The reliability of hardware lies completely in the choice of components, circuit protection and in the design, manufacture and assembly of the PCB, which is the electromechanical hardware support. That is why international standards should be used for the electronic system design. These standards define PCB's development details, such as the Association Connecting Electronics Industries (IPC) standards and the European Cooperation for Space Standardization (ECSS), which provide industrial criteria to evaluate the quality of the designs under a single frame of reference. As following, some guidelines are recommended to be applied for the design of high-quality and reliability systems in space environments:

- Design Standard for Rigid Printed Circuit Boards and Assemblies (MSFC-STD-3425). In this standard, two documents of the IPC are named, the IPC-2221A and
IPC-2222; the first talks about the general requirements for the design of PCBs and the second sets specific details over the designs for the installation and connection of components, assets and liabilities, using the 3 -class performance for high-reliability electronic products.

- Design Rules for Printed Circuit Boards (Q-ST-70-12C) is an ECSS document that specifies rules for the design of printed circuit boards.

Another important issue to consider is that of ruptures of welding joints, whether by mechanical vibrations or difference between the coefficient of thermal expansion (CTE) of the components and the PCB. To decrease the rupture probability, the gap between the thermal expansion coefficients of the components and the board must be reduced, so that both will behave in a similar way at strong temperature changes.

To give greater rigidity to the devices against mechanical vibrations, the "footprints" of the components should be over dimensioned with respect to the physical size of the component, in order to apply greater volume of welding and give better mechanical support. The dimensions are given by the standard Naming Convention for Standard SMT Land Patterns (IPC 7351B), where the design parameters of the components footprints are established according to the application. In this case, it is used Level A for "Most Material Condition" for high-shock and vibration applications. In addition, physical supports should be added, which hold the components during periods of extreme vibration; the standard Class 3 High-Reliability Applications (IPC-2221) - section 8.1.9.2 mentions the criteria for selection of these supports.

Commercial off the shelf (COTS) devices used in CubeSat systems are not designed to operate in hostile environments such as space; that is why it is recommended to use a protective covering or conformal coating, to give extra protection against extreme changes in temperature and degassing (Hardy, 2011). According to military specification MIL-I-46058C, there are several types of conformal coatings which highlight the XY type, also called paraxylylenes, that offer protection to extreme temperature ranging from $-200^{\circ} \mathrm{C}$ and $150^{\circ} \mathrm{C}$ and high tolerance to radiation. In addition, its implementation by vacuumdeposition process reduces the possibility of bubbles trapped under the overlay, which, at low pressures, may compromise the uniformity of the cover. The use of the paraxylylen Parylene D coating for CubeSat applications is recommended, since it maintains their physical and electrical properties at high temperatures. According to the standard MIL-I-46058C, a 
layer of $0.6 \mathrm{~mm}$ of thickness of this coating fully covering all the components can offer a substantial increase in tolerance, without adding considerable weight.

\section{INTERNATIONAL TRAFFIC IN ARMS REGULATIONS RESTRICTIONS}

In order to increase the reliability and damage tolerance of the satellite's hardware, ideally it must use components designed to operate in harsh environments, as the high radiation tolerance components also known as Rad Hard; however, the exportation of much of these components is controlled by the International Traffic in Arms Regulations (ITAR) (22 CFR 120 through 130), making it difficult to purchase in the market.

\section{COMPONENT SELECTION FOR A CUBESAT REMOTE SENSING SYSTEM SELECTION OF OPTICAL SENSOR FOR IMAGE CAPTURE}

Image sensors are an array of light sensitive photosites, which quantify the amount of received light energy. An image is made up by organizing the values collected from each photosite on a matrix of pixels. There are currently two available technologies for image sensors, charge-coupled device (CCD) and CMOS.

The CCD technology consists of a matrix of metal-oxidesemiconductor (MOS) capacitors doped p-type, in which each capacitor builds up an electrical charge proportional to the perceived light intensity; then, an internal circuit transfers its charge to a neighbor in the same row in only one direction, the last pixel of the line delivery that burdens an operational amplifier, which is responsible for converting it to voltage. This results in an analog signal from each line of the matrix which is then sampled to digitize it (Litwiller, 2009).

A CMOS sensor is an active pixel sensor (APS) sensitive to visible light. It makes use of a digital signal processing (DSP) in an embedded system, to process the data of intensity that perceives each cell of the pixel matrix, recreating an image. This kind of sensor is known as CMOS since it uses technology based on metal-oxide-semiconductor field-effect transistors (MOSFET); each of its photosites contains an amplifier for electrical signal and usually includes a digital converter in the own chip (Litwiller, 2009).

To capture multispectral color images, usually an array of Bayer RGB filters is placed between the lens and the photodiode, to prevent the passage of all frequencies of light except for the bands of red, blue and green; with them, the RGB color model can be calculated and a good approximation of the visible scene to the human eye can be composed (Litwiller, 2009).

Table 2 shows a comparative analysis between the image sensor technologies, the CMOS and CCD, showing relevant characteristics useful for the images' capture process.

Table 2. CCD versus CMOS

\begin{tabular}{|c|c|c|}
\hline Characteristics & CCD & CMOS \\
\hline Dynamic range & Yes & No \\
\hline Response capability & No & Yes \\
\hline Reliability & Yes & Yes \\
\hline Energy consumed & No & Yes \\
\hline Size & No & Yes \\
\hline Capture time & No & Yes \\
\hline Blooming & No & Yes \\
\hline Windowing & No & Yes \\
\hline Temperature & No & Yes \\
\hline
\end{tabular}

The dynamic range is the ratio between the saturation level and noise in an image; since the CCD does not integrate the digitalization of the signal into the sensor, this process is carried out in specialized systems, which can get a lower level of noise through the use of DSP.

The response capability is the parameter that measures the ratio between the output voltage of the sensor and a certain incident light energy density; this is expressed in electric potential $(\mathrm{V})$ by energy $(\mathrm{J})$ in the unit of area $\left(\mathrm{cm}^{2}\right)-\left(\mathrm{V} / \mu \mathrm{J} \mathrm{cm}^{2}\right)$. CMOS technology has amplifiers that increase the profit by an integrated circuit, improving sensitivity to light in each pixel. In terms of reliability, both technologies have similar behaviors, so one cannot be considered as better than the other.

In the case of the power and size, CMOS has a clear advantage by integrating the required embedded system on a single chip to obtain the images, while CCD requires extra components. Additionally, the polarization voltage increases considerably for CCD technologies, as it uses voltages around 12 to $15 \mathrm{~V}$, while CMOS uses voltages from 2.8 to $5 \mathrm{~V}$. In terms of the speed of frame capture or capture time, it is similar in both technologies; nevertheless, CMOS presents the advantage of integrating all the capture process on a single chip, so power and data signals travel a shorter distance, improving propagation times and reducing the inductance.

A phenomenon to be considered in RS systems is blooming, that is an effect of overflowing of charge in a pixel due to the 
excess of incident light, resulting in a contribution of charge to neighboring pixels. CMOS technology presents an independent design, where each photosite delivers digital data separately to avoid blooming effect, while CCD sensors do not have this feature, making them more sensitive to this effect. The windowing is a feature added to CMOS sensors, where one can choose to capture only a fraction of the frame (picture capture), saving time and energy. This functionality is not implemented in the CCD sensors. Finally, the operating temperature represents a big problem in the CCD as it increases their sensitivity to noise, to the point of doubling it every $7^{\circ} \mathrm{C}$. Therefore, they require to be refrigerated for space applications (Litwiller, 2009; Eastman Kodak Company, 2009).

\section{SELECTION OF THE CONTROLLER FOR THE IMAGE SENSOR CONTROL SYSTEM}

The control system must have communication interfaces with the CMOS sensor and with external memories in order to capture and storage the images. Different microcontroller systems that fill these requirements are presented in Table 3.

From the analyzed microprocessors, it was selected the STM32F407 of architecture ARM Cortex-M4, which possesses the digital camera interface (DCMI) for control and data transmission from the image sensor and flexible static memory controller
(FSMC) module; it also has a DMA module to transfer data at high speed between peripherals and RAM memories. All these modules are implemented in hardware, which is configured from the CPU and runs concurrently to this, leaving the processor free for other operations. Additionally, this architecture of microcontrollers handle floating point logic, which optimizes it for DSP analysis. It adds special instructions that facilitate the handling and processing of images (ARM, 2013). Figure 3 shows the block diagram that describes the process for the image's capture and storage.

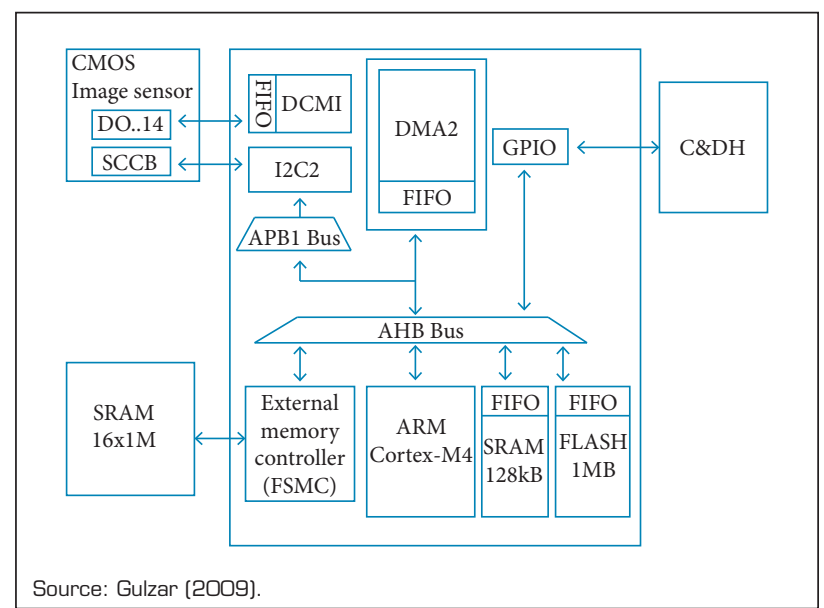

Figure 3. Block diagram of the controller of the image acquisition system.

Table 3. Comparison of microcontrollers.

\begin{tabular}{|c|c|c|c|c|c|c|c|}
\hline DSP & $\begin{array}{c}\text { Maximum } \\
\text { frequency }(\mathrm{MHz})\end{array}$ & Manufacturer & FSMC & DCMI & DMA & I/O interface & $\begin{array}{c}\text { Program memory } \\
\text { (KB] }\end{array}$ \\
\hline Tms320dm643 & 700 & $\mathrm{TI}$ & $\mathrm{Si}$ & $\mathrm{Si}$ & $\mathrm{Si}$ & $\begin{array}{c}\text { I2C } \\
\text { McBsp } \\
\text { SPI } \\
\text { CAN } \\
\text { PCI } \\
\text { Ethernet }\end{array}$ & $\begin{array}{l}\text { No integrated } \\
\text { memory }\end{array}$ \\
\hline Tms320vc5509a & 200 & TI & $\mathrm{Si}$ & $\mathrm{Si}$ & $\mathrm{Si}$ & $\begin{array}{c}\text { USB } \\
\text { Full-Speed } \\
\text { McBSP }\end{array}$ & 64 \\
\hline OMAP3515 & 600 & TI & $\mathrm{Si}$ & $\mathrm{Si}$ & $\mathrm{Si}$ & $\begin{array}{c}\mathrm{MMC} / \mathrm{SD} \\
\mathrm{POP} \\
\mathrm{I} 2 \mathrm{C} \\
\text { UART } \\
\text { USB }\end{array}$ & 32 \\
\hline ADSP-BF561 & 600 & Analog devices & $\mathrm{Si}$ & $\mathrm{Si}$ & $\mathrm{Si}$ & $\begin{array}{c}\text { IrDA } \\
\text { UART } \\
\text { Ethernet }\end{array}$ & 328 \\
\hline STM32F407 & 168 & STMicro & $\mathrm{Si}$ & $\mathrm{Si}$ & $\mathrm{Si}$ & $\begin{array}{c}\text { I2C } \\
\text { CAN } \\
\text { USART } \\
\text { USB } \\
\text { MMC/SD }\end{array}$ & $\begin{array}{l}\text { Flash: } 192 \\
\text { SRAM: } 64\end{array}$ \\
\hline
\end{tabular}




\section{SELECTION OF THE MEMORIES FOR STORING IMAGES}

Table 4 presents a comparative study between different types of volatile and non-volatile memories, in order to identify which of them is best suited for RS system, taking into account main operations, as the image capture and storage, and considering fundamental requirements as a low energy consumption and the available volume.

Due to the amount of data and the flow speed of information that the sensor delivers, the selection criteria of the memory are its speed and capacity. In order to avoid overloading in the internal RAM of the microcontroller, the system needs a fast enough memory and its capacity for data storage must be enough to allow staying in it a burst of images. Then, commercially available memory options are evaluated in order to select which is the one that is better suited to a RS system on-board a CubeSat.

SRAM memories are used as cache memory in personal computers since they offer faster speeds of reading and writing (8 ns) than all other memories; the SRAM, however, can store information when the system is energized. Once the power source is removed, the information will be lost. A dynamic random access memory (DRAM) is superior to the SRAM in terms of density, i.e. it has greater volume of data. However, it increases the time of reading/writing. SRAM memories, being the fastest but volatile, require a secondary storage to allow the download of images at any time.

After obtaining the images, RS system enters a wait state until it receives the command to send an image. In order to minimize the cost of energy in this state, the system should enter low consumption by disabling the peripherals, so it is necessary to store the images in a non-volatile memory for later transmission. There are three types of non-volatile memory technologies: Flash memory, ferro-electric random access memory (FRAM) and magnetic random access memory
(MRAM). Flash memory is considered more suitable for satellite data transmission's needs for the following reasons:

- Flash memories can achieve the greatest amount of information per unit area, since a flash cell is made up of just one transistor, while the FRAM and MRAM cells need a transistor, a capacitor, or an union of magnetic tunnel, which implies greater physical space for the same amount of information (Semiconductor Industry Association, 2014; Kim et al., 2002).

- $\quad$ Flash memories have the property of saved multi-bit per cell, which increases the density of the memory, reducing the cost per bit (King, 1999).

- The manufacturing process of the flash memory is compatible with current CMOS processes and is a sustainable solution for embedded system applications. A flash memory cell is a MOSFET; the rest of non-volatile memories require the integration of other materials that are not compatible with conventional CMOS processes, which makes them less reliable for integration with other analog or logical devices (She, 2003).

\section{IMAGE CAPTURE PROCESS}

Three stages for the acquisition of images are defined within embedded software: the first is the moment of image capture, followed by in-memory main storage and, finally, a stage of images saved in non-volatile memory.

At the moment of image's capture, the sensor loads their cells according to the intensity of light that receives and delivers this information as digital values organized as a Bayer matrix to the DSP. This data is processed to generate the delivery format (RAW-RGB, RGB or YUV) image, which is made by hardware and is called exposure time. CMOS image sensors are progressive scanners, i.e. they determine the amount of incident light at

Table 4. Memory technologies comparison.

\begin{tabular}{|c|c|c|c|c|c|c|}
\hline Memory & DRAM & SRAM & Flash-NOR & Flash-NAND & FRAM & MRAM \\
\hline Volatile/Non volatile & Volatile & Volatile & Non volatile & Non volatile & Non volatile & Non volatile \\
\hline Reading method & Destructive & Partially destructive & No destructive & No destructive & Destructive & Destructive \\
\hline Reading voltage & $\sim 1 \mathrm{~V}$ & $\sim 1 \mathrm{~V}$ & $2 \mathrm{~V}$ & $2 \mathrm{~V}$ & $1.5 \mathrm{~V}$ & $3.3 \mathrm{~V}$ \\
\hline Writing voltage & $\sim 1 \mathrm{~V}$ & $\sim 1 \mathrm{~V}$ & $10 \mathrm{~V}$ & $18 \mathrm{~V}$ & $1.5 \mathrm{~V}$ & $3.3 \mathrm{~V}$ \\
\hline Reading speed & $8 \mathrm{~ns}$ & $8 \mathrm{~ns}$ & $60 \mathrm{~ns}$ & $60 \mathrm{~ns}$ & $80 \mathrm{~ns}$ & $30 \mathrm{~ns}$ \\
\hline Writing speed & $50 \mathrm{~ns}$ & $8 \mathrm{~ns}$ & $1 \mathrm{us}$ & $1 \mathrm{~ms}$ & $80 \mathrm{~ns}$ & $50 \mathrm{~ns}$ \\
\hline Biggest vector built & & & $2 \mathrm{~Gb}$ & $512 \mathrm{~Gb}$ & $64 \mathrm{Mb}$ & $16 \mathrm{Mb}$ \\
\hline
\end{tabular}

Source: She (2003). 
discrete moments of time and discharge their voltage values whenever they deliver data; it is for this reason that CMOS sensors do not require a shutter to regulate the exposure time, since this is already determined by the manufacturer because it depends on the size of the frame and the speed of the internal DSP. This value is measured in frames per second (fps) and commercially it is between 580 and 3 fps.

The second stage consists on the image storage in the main memory. The DCMI, which is a synchronous parallel interface capable of receiving streams of data at high speeds from up to 14-bit CMOS camera module, is used to receive an image from the embedded system.

Figure 4 shows the block diagram of the DCMI interface. Signals DCMI_PIXCLK, DCMI_D[0:13], DCMI_HSYNC and DCMI_VSYNC are generated by the sensor; these serve as the control of transmission of the image. The data is stored in a 32-bit buffer that when it gets filled up, it sends a request to the DMA, which flushes the data from the DCMI buffer to a previously configured memory location. The DMA is used to provide data transfer at high speed between peripherals and memories, without action of the CPU. This module combines a dual master bus (AHB) with a separate first input-first output (FIFO) buffer architecture that optimizes the system bandwidth and improves response times.

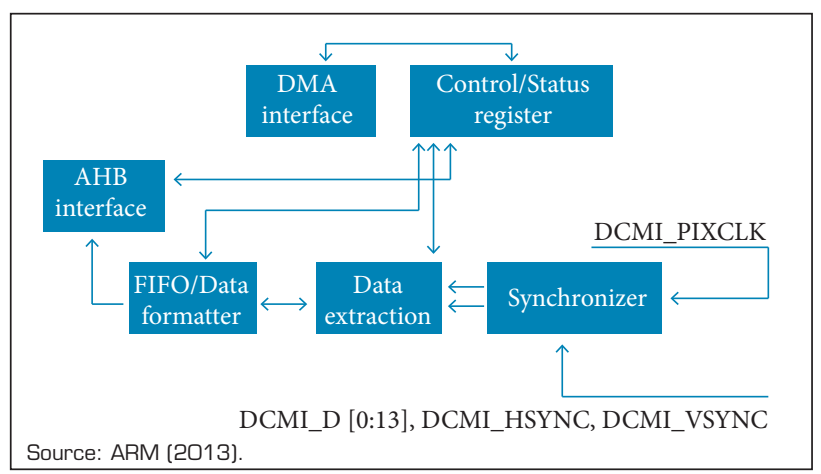

Figure 4. DCMI block diagram.

The DMA manages requests for peripherals and starts a transfer whenever one is received; a DMA transfer is the flush of data from the peripheral directly to a space in memory, where it will remain while the power supply is not interrupted. High-speed memories, as the SRAM access, require the use of the flexible static memory control (FSMC) controller that acts as an interface for synchronous and asynchronous memories, translating the AHB transactions to the appropriate external reading or writing protocol (ARM, 2013).
The last stage consists of the non-volatile memory image storage, where it will remain until being required by the $\mathrm{C} \& \mathrm{DH}$. The access to non-volatile memory is also performed by the module DMA, using memory-to-memory transfer mode. To counteract the effects of SEE, a checksum field is recommended to save the image to verify the consistency of the data when the $\mathrm{C} \& \mathrm{DH}$ receives a transmission request from Earth.

\section{THE SATELLITE IMAGE SENSOR DATA PROCESSING}

As mentioned previously, the CMOS sensor delivers information as a Bayer array with raw data and the size of $\mathrm{n} x \mathrm{~m}$. The Bayer array consists of three color components: green, red and blue; it applies to this information an algorithm of color interpolation or rebuilding, which is a digital process used to reconstruct an image to the RGB color model from samples acquired from the multispectral CMOS image sensor (Adams et al., 1998).

There are several rebuilding algorithms to calculate RGB color model from the Bayer array; among the most prominent ones, there are: Pixel Binning, Nearest Neighbor, Bilinear, Smooth Hue Transition and Edge-sensing Bilinear (Elizondo and Maestre, 2005). However, when using a Bayer to RGB conversion algorithm, the dimensions of the image are increased from ( $\mathrm{nx} \mathrm{m}$ ) to 3 matrices of the same size, that is ( $\mathrm{n} \times \mathrm{mx}$ ). This is why it is recommended that the information delivered by the CMOS sensor be transmitted without being processed, so power consumption is reduced by having less amount of information in the queue.

\section{DEVELOPMENT OF A REMOTE SENSING SYSTEM FOR A 3U CUBESAT}

Taking into account the issues due to the radiation of highenergy particles, low atmospheric pressure, thermal shocks, vibration during the launch and low processing capacity — and according to the recommendations presented in the previous sections -, the software and hardware design of a RS system for CubeSat space applications are shown as follows.

\section{DEVELOPMENT OF THE REMOTE SENSING SYSTEM CONTROL SOFTWARE}

The available functionalities must be specified for the design of the RS system control software, which is made through the use of a case diagram that describes the driver in terms of the processes that runs each actor. At the same time, the actors represent subsystems or external devices that interact with the 
software. At first, the software operation environment must be established, identifying internal and external subsystems that will be in contact with the RS system. In this case, the master will be the central computer of the satellite $(\mathrm{C} \& \mathrm{DH})$ and the payload driver is represented as an intern actor who executed the orders received from the master.

Figure 5 shows the minimum capabilities that the C\&DH should carry out in the RS system; among them, there is the option to configure the camera in low power consumption when no actions are required. The other use cases are directly related to the process of image acquisition. In this case, one for ordering a capture and another for asking for the image transmission. The C\&DH should be able to define the parameters to take the photo, like frame size, subsampling of the image, the gain and the delivery format. Another function that must be available is to be able to request the state of the camera's memories in order to verify if there is space left to store more images.

Next, the deployment of processes is explained, which defines the behavior of the control software. The activity diagram in

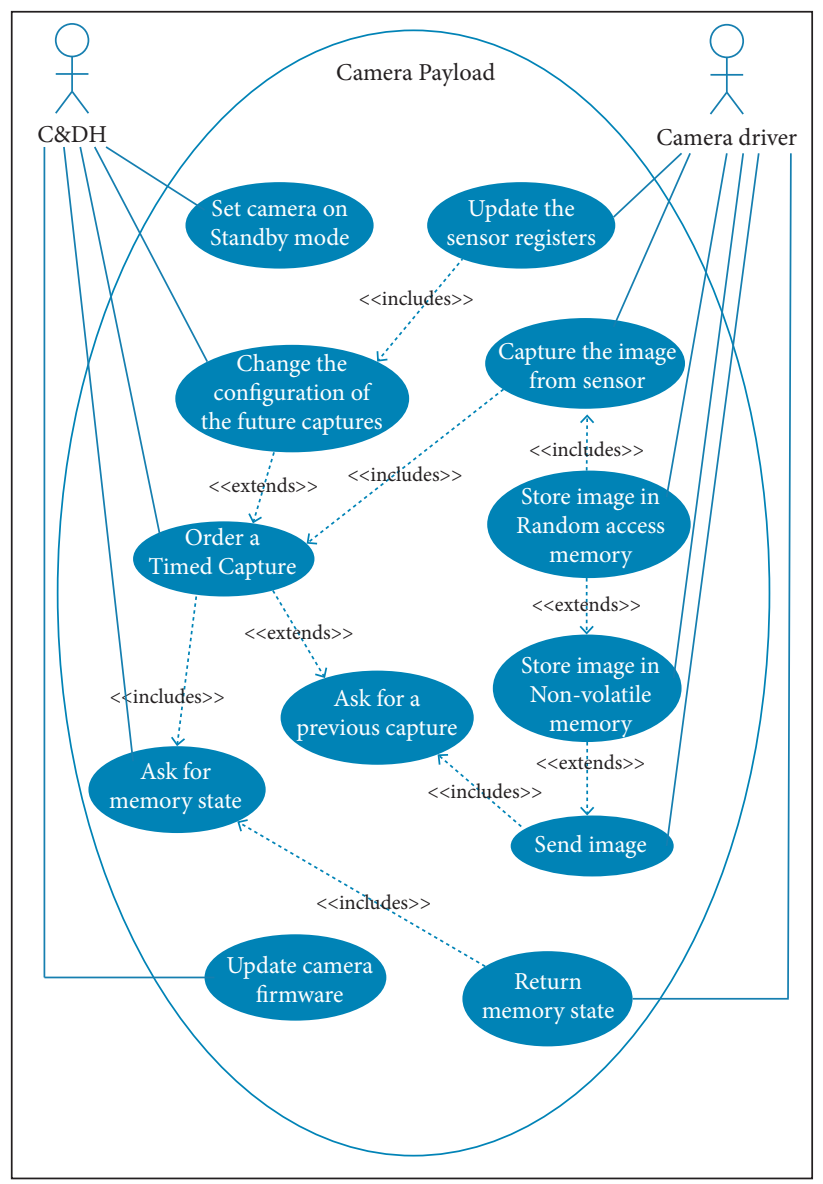

Figure 5. Use case diagram of the RS system software.
Fig. 6 represents the time for execution of each task involved in the image's capture.

The "Command_Handler" task that runs in the background is a persistent and high priority task, which is on charge of reception and administration of the commands sent by the $\mathrm{C} \& \mathrm{DH}$. When a capture command is received, it will set a timer to run the capture task at the proper time; this sequence corresponds to the steps to capture an image. During this task, the DMA module is activated, so that it will be attentive to the requests the DCMI peripheral could send, thus activating the DCMI and, as soon as it reaches a start of frame indicated by the VSYNC signal, the DMA will start the flush of data to the principal RAM memory.

As soon as the DCMI and DMA modules are activated, the sensor data will be flushed by hardware. The Capture_Finished method must set off the DCMI, so that the received data may remain static while it is copied to the external flash memory; the DCMI, however, is not disabled until it ends receiving the frame. So, in order to disable the DMA, a recurrent query must take place, asking if it has already finished the image capture, otherwise it can cause image damages.

After the DMA and DCMI modules are off, the software executes the routine of Persistent_Save. In this function, the DMA is used to flush the data from main memory (SRAM) to non-volatile memory (flash). Additionally, it adds the image metadata to the delivered package; for example, the values of gain and the average luminance. Finally, it turns a flag indicating

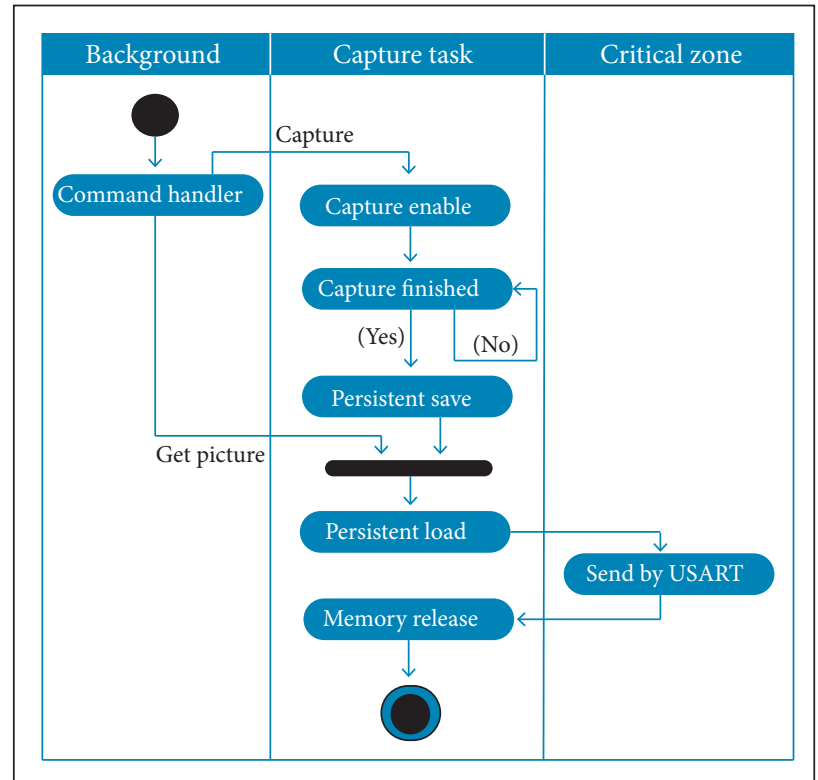

Figure 6. Activity diagram for tasks 
that an image has been taken correctly and it is waiting to be transmitted.

Once an image is captured and stored, C\&DH may submit the request for that image. When this command is received, the first flush of data will be initiated from the flash memory to the main memory through DMA, which must be reconfigured in the Persistent_Load method.

In order to send the image through serial port, the system interruptions should be disabled. For this, one must run the routine in a safe area of the code, to ensure the integrity of the data. While the interruptions are disabled, the system loses the ability to listen to the commands sent by the C\&DH, so the use of this safe area should be limited to the transmission of images through the USART interface, with the Send_By_ USART method. Many of the tasks of the camera driver have to run at specific times or even at the same time; this is why it is recommended to use a real time operating system (RTOS).

Radiation and other sources of external interference can cause critical errors in the software, such as corruption of the code, change in the data and unscheduled resets. Code and data corruption can be permanent or temporary. For permanent errors, the only solution available is to update the system's control software. Therefore, it is recommended to store versions of all the firmware of the subsystems in the C\&DH.

All captured images must be transferred to a special memory region in the SRAM that can contain a complete image of 8 bits per pixel; the other part of the memory will store image compressions, cuts and other useful data for later use. Images are saved in flexible image transport system (FITS) format, that includes the following metadata in the header: exposure, capture time, temperature sensor, firmware version, and commentary on the current camera mode and some settings such as resolution and gain parameters (Pence, 2010).

\section{DEVELOPMENT OF THE RS SYSTEM HARDWARE}

Some considerations for the manufacture and assembly of RS system hardware are presented as following, implementing the design recommendations proposed in the section: Considerations for the design of remote sensing systems of deployment in the Low Earth Orbits.

\section{Device's Footprints for High-Reliability Applications}

For the design of the land pattern, it is recommended a specialized software tool like IPC-7351 LP Wizard ${ }^{\circledR}$ Mentor Graphics or "PCB Library Expert ${ }^{\circledR}$, which has the ability to design land patterns with the specifications dictated by the standard IPC-7351B level A "Very Robust Solder Joint" for high reliability class 3 applications.

In the case of ball grid array (BGA) devices, the solder mask in the area around the copper pad must be removed to decrease the probability of the appearance of cracks into solder joints (Fig. 7a), due to the high mechanical stress that can cause contact between the mask and solder. This reduction of the mask allows the solder to stick around the pad, improving contact with the surface and increasing union's reliability (Fig. 7b).

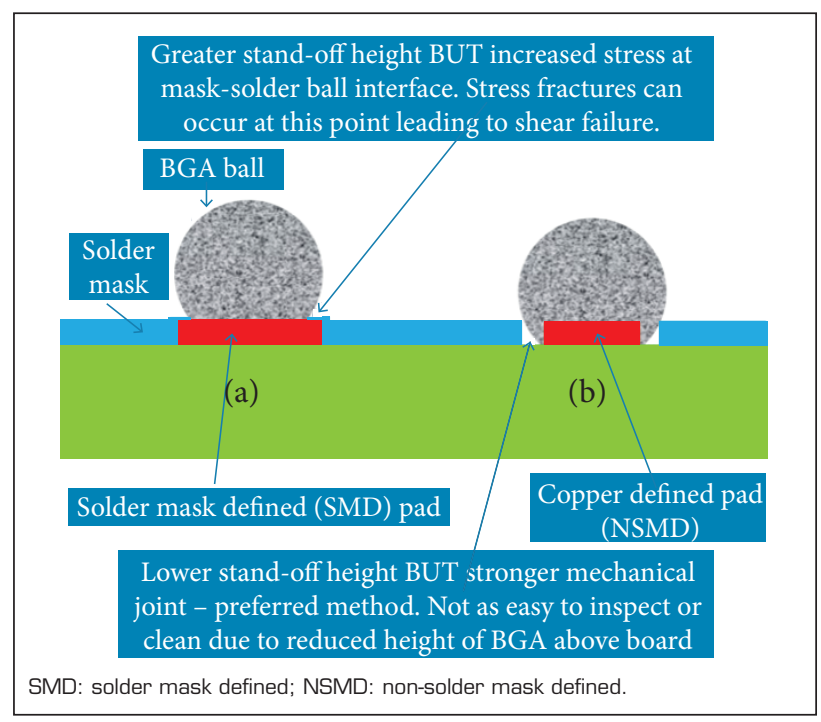

Figure 7. Definition of the BGA pad (Johnson, 2014). (a) BGA copper pad with solder mask around; (b) BGA copper pad without solder mask around.

\section{Electromagnetic Compatibility in the Design}

One of the most important aspects in the electronics equipment is the property of operating without causing interference to neighboring subsystems; there are different considerations that can be applied in the design of the PCB for better performance in electromagnetic compatibility (EMC). One must be sure to minimize the area of the loop, not to place circuits between connectors, control the transition time from digital signals and provide return signal plane (Hubing, 2003).

Another aspect to consider is electromagnetic interference (EMI) shielding signals. This can be done routing them between the power and ground planes. So, in the PCB design, it must consider the use of multiple layers to provide extra protection to the internal planes and make signals less likely to interact with external interference sources. 
In the PCB design, the aspect ratio must not exceed 8:1 to prevent break in any via due to a mechanical stress (Marshall Space Flight Center, 2006). In addition to axial-leaded components with a weight equal or greater than $5 \mathrm{~g}$ per lead (leg), they must be secured to the PCB with mechanical stands and not only solder joints. It is advisable to assemble the PCB under the standards IPC j-STD-001 class 3 and IPC-A610 class 3.

\section{Solder Joints for Space Applications}

For space applications, the use of lead-based solder is allowed, which can prevent problems as delamination, rupture of internal paths and blistering, due to its lower melting point in comparison with the lead-free solder (Kessel, 2005). In the space applications electronic hardware addendum to the IPC-J-STD-001D standard described by IPC, titled as "Requirements for Soldered Electrical and Electronic Assemblies", it is specified that the solder alloys that can be used in space applications should be one of the following: Sn60Pb40, Sn62Pb36Ag2, Sn63Pb37 or Sn96.3Ag3.7 (Association Connecting Electronics Industries, 2008).

Table 5 shows the properties of the different solder alloys mentioned by IPC-J-STD-001D, whereas all solders have a similar behavior in the majority of its properties; it is used melting point and tensile strength parameters as criteria for solder selection. As seen in Table 4, the Sn62Pb36Ag2 presents the lower melting point, so it is less likely to damage the components by heat excess at the assembly time. It has, however, less resistance to mechanical stress. Moreover, Sn96.3Ag3.7 alloy presents the greater tensile strength. This indicates the maximum stress that can withstand before breaking the solder, which makes it more reliable to vibration, but with regard to its melting point, high value could affect components in their assembly process. For these reasons, it is recommended to use $\mathrm{Sn60Pb} 40$ solder or $\mathrm{Sn63Pb37}$, since they offer the best relation between resistance to mechanical stress and fusion temperature, in addition to GomSpace used in the development of its prototype RS system for CubeSats, the NANOCAM-1U (GomSpace, 2001).

\section{Printed Circuit Board Material for Vacuum Applications}

The material selected must comply with the ASTM-E-595 standard, which specifies that the materials must not exceed $1 \%$ in "Total Mass Loss" (TML) and $0.1 \%$ in "Collected Volatile Condensable Materials" (CVCM) in vacuum testing. Table 6 shows a list of materials and their results in the vacuum test conducted by NASA, collected in the database "Outgassing Data for Selecting Spacecraft Materials" of this entity which meet the above mentioned requirements.

The type of material that one chooses affects the reliability of the system. The standard IPC-4101 describes the specifications that must comply with the materials to be used in PCB's rigid multilayer; some of the most important properties to be considered in the choice of the PCB materials are the dielectric constant for a better signal integrity, a greater transition glasses (Tg) and a lower CTE, allowing thermal stability against temperature cycles.

According to the standard ECSS-Q-ST-70-12c, the most used materials in the PCB's manufacture for space applications are the

Table 5. Solder alloys (Association Connecting Electronics Industries, 2006).

\begin{tabular}{|c|c|c|c|c|c|c|c|}
\hline Solder alloy & $\begin{array}{l}\text { Melting point } \\
{\left[{ }^{\circ} \mathrm{C}\right] \text { solid/liquid }}\end{array}$ & $\begin{array}{l}\text { Density } \\
{\left[\mathrm{g} / \mathrm{cm}^{3}\right]}\end{array}$ & $\begin{array}{c}\text { Electrical } \\
\text { resistivity } \\
\text { [ohm-m] }\end{array}$ & $\begin{array}{l}\text { Thermal } \\
\text { conductivity } \\
\text { [W/m-K] }\end{array}$ & $\begin{array}{c}\text { Tensile strength } \\
\text { at break } \\
{\left[\mathrm{Kgf} / \mathrm{cm}^{2}\right]}\end{array}$ & $\begin{array}{c}\text { Tensile } \\
\text { elongation at } \\
\text { break [\%] }\end{array}$ & $\begin{array}{c}\text { Brinell } \\
\text { hardness } \\
\text { [HB] }\end{array}$ \\
\hline $\mathrm{Sn} 60 \mathrm{~Pb} 40$ & $183 / 191$ & 8.50 & 0.153 & 49 & 535 & 40 & 16 \\
\hline $\mathrm{Sn} 63 \mathrm{~Pb} 37$ & $183 / 183$ & 8.40 & 0.145 & 50 & 525 & 37 & 17 \\
\hline Sn96.3Ag3.7 & $221 / 221$ & 7.37 & 0.123 & 55 & 580 & 35 & 15 \\
\hline Sn62Pb36Ag2 & $179 / 179$ & 8.41 & 0.145 & 50 & 490 & -- & 16 \\
\hline
\end{tabular}

Table 6. Results of outgassing tests \% TML, \% CVCM for PCB materials.

\begin{tabular}{|c|c|c|}
\hline Material & \% TML & \% CVCM \\
\hline CER-10 Glass/PTFE/Ceramic laminate material & 0.02 & 0.01 \\
\hline FR406, Woven e-glass, Copper foil & 0.43 & 0.00 \\
\hline Alumina, Gold, PD/AG & 0.02 & 0.00 \\
\hline Circuit board composite Nema G10 Mica/CE1155 & 0.48 & 0.01 \\
\hline G10 Fiberglass per MIL-I-24768/27 & 0.30 & 0.00 \\
\hline Laminate 602 Teflon/Fiberglass W/O Copper & 0.01 & 0.00 \\
\hline
\end{tabular}


FR4 and polyimide. The last one could have a Tg greater than $250^{\circ} \mathrm{C}$ and it would be more stable to heat than the FR4, which has a Tg up to $180^{\circ} \mathrm{C}$. Among the commercial polyimides, the $35 \mathrm{~N}, 85 \mathrm{~N}$ and $85 \mathrm{NT}$ of Arlon are recommended, which present good behavior regarding the dielectric constant, coefficient of thermal expansion and $\mathrm{Tg}$.

\section{CONCLUSIONS}

In this paper, the critical elements related to the operating environment were identified, which are decisive in the development of an embedded system to capture and storage satellite images, such as: thermal radiation, particle radiation, outgassing, and some ways to mitigate these effects.

A software/hardware architecture was proposed for the implementation of a system for capturing satellite images, and the capturing and storing process of an image taken with a CMOS sensor was established.

A software controller in real time was structured, with features of critical system for the control and management of the information of a CMOS sensor, detailing the capture sequence and storage techniques in memory arrays.

Finally, some parameters, materials and coatings were identified, which could be used in the PCBs manufacture for aerospace operation.

\section{FUTURE WORK}

It is expected the design of the optical RS system, according to the restrictions of the CubeSat standard, with emphasis on lenses and filters for an aerospace operation environment.

In addition, it is expected to include compression algorithms with and without loss in the proposed software controller, in order to transmit a mosaic to the ground station and then select the best for download in original format.

Besides, it is planned to implement and perform protoflight tests in laboratories specialized in aerospace systems, to verify the correct operation of the developed prototype, including a LEO thermal cycle emulation, as well as the observation of the material's behavior in vacuum.

\section{REFERENCES}

Adams, J., Parulski, K. and Spaulding, K., 1998, "Color processing in digital cameras", IEEE Micro, Vol. 18, No. 6, pp. 20-30. doi: $10.1109 / 40.743681$

Alminde, L., Bisgaard, M., Vinther, D., Viscor, T. and Ostergard, K., 2003, "Educational Value and Lessons Learned from the AAUCubeSat project", Proceedings of the International Conference Recent Advances in Space Technologies, Istanbul, Turkey.

ARM, 2013, "ARM ${ }^{\circledR}$ Cortex ${ }^{\mathrm{TM}}-\mathrm{M} 4$ Processor Technical Reference Manual", ARM Limited, USA.

Association Connecting Electronics Industries (IPC), 2008, "Requirements for soldered electrical and electronic assemblies - IPC J-STD-001D", Bannockburn, Illinois, USA.

Burlyaev. D. and van Leuken, R., 2014, "Systemfault-Tolerance Analysis of COTS-Based Satellite On-Board Computers", Microelectronics Journal, Vol. 45, No. 10, pp. 1335-1341. doi:10.1016/j. mejo.2014.01.007

Eastman Kodak Company, 2009, "Solid State Image Sensors Terminology", Microelectronics Technology Division, Rochester, USA.

Elizondo, J.E. and Maestre, L.P., 2005, "Fundamentos de Procesamiento de Imágenes", Universidad Autónoma de Baja California, México.

Enokuchi, A., Nagai, M., Funase, R., Nakamura, Y. and Nakasuka, S., 2015, "Remote Sensing by University of Tokyo's Pico-Satellite Project 'PRISM'", Department of Aeronautics and Astronautics, University of Tokyo, Retrieved in Jan 06, 2015, from http://www.dlr.de/ Portaldata/49/Resources/dokumente/archiv5/0605_Enockuchi.pdf
Fayazbakhsh, K. and Abedian, A., 2010, "Materials Selection for Applications in Space Environment Considering Outgassing Phenomenon", Advances in Space Research, Vol. 45, No. 6, pp. 741749. doi:10.1016/j.asr.2009.11.017

Funase, R., Takei, E., Nakamura, Y., Nagai, M, Enokuchi, A., Yuliang, C., Nakada, K., Noriji, Y., Sasaki, F., Funane, T., Eishima, T. and Nakasuka, S., 2006, "Technology Demonstration on University of Tokyo's Pico-Satellite 'XI-V' and its Effective Operation Result Using Ground Station Network", Acta Astronautica, Vol. 61, No. 7-8, pp. 707-711. doi:10.1016/j.actaastro.2006.12.032

Jernes, N, 2011, "NanoCam C1U Datasheet. GS-DS-NANOCAM-6.2, Issue: 6". Gomspace, Aalborg East, Denmark.

Greenland, S. and Clark, C., 2010, "CubeSat Platforms as an On-Orbit Technology Validation and Verification Vehicle", Retrieved in January 27, 2015, from http://www.clyde-space.com/documents/1805

Grossman, E. and Gouzman, I., 2003, "Space Environment Effects on Polymers in Low Earth Orbit", Nuclear Instruments and Methods in Physics Research Section B: Beam Interactions with Materials and Atoms, Vol. 208, pp. 48-57. doi:10.1016/S0168583X(03)00640-2

Gulzar, K., 2009, "Camera Design for Pico and Nano Satellite Applications", Department of Space Science, Kiruna, Switzerland.

Hardy, A., 2011, "Parylene Coating Can Protect COTS Electronics in Aerospace and Defense Applications", Retrieved in Jan 06, 2015, from http://www.militaryaerospace.com/articles/print/volume-22/ 
issue-30/opinion/parylene-coating-can-protect-cots-electronics-inaerospace-and-defenes-applications.html

Hubing, T., 2003, "PCB EMC Design Guidelines: a Brief Annotated List", IEEE International Symposium on Electromagnetic Compatibility, Vol. 1, pp. 34-36. doi: 10.1109/ISEMC.2003.1236559

Johnson, A., 2014, "Prenier EDA Solutions", Retrieved in Jul, 2014, from http://www.eda.co.uk/design_community/design_tips/soldermask.html

Kessel, K., 2005, "NEPP TRO - Lead-Free Soldering for Space Applications Lead-Free Solder Body of Knowledge", NASA, Kennedy Space Center, ITB, Inc., Dayton, USA.

Khurshid, K., Mahmood, R. and ul Islam, Q., 2013, "A Survey of Camera Modules for CubeSats-Design of Imaging Payload of ICUBE-1", Proceedings of the 6th International Conference on Recent Advances in Space Technologies (RAST'13), Istanbul, Turkey.

Kim, D.W., Prins, F.E., Kim, T., Kwong, D.L. and Banerjee, S., 20०2, "Charge Retention Characteristics of SiGe Quantum Dot Flash Memories", Proceedings of the Device Research Conference, USA.

King, Y.C., 1999, "Thin Dielectric Technology and Memory Devices", University of California, Berkeley, USA.

Knight, J.C., 2002, "Safety Critical Systems: Challenges and Directions", Proceedings of the 24rd International Conference on Software Engineering, Orlando, USA.

Kuuste, H, 2012, "Estcube-1 Tether End Mass Imaging System Design and Assembly", Institute of Physics Information Technology, Faculty of Science and Technology, University of Tartu, Tartu, Estonia.

LaBel, K.A., Gates, M.M., Moran, A.K., Marshall, P.W., Stassinopoulos, J.B., Seidleck, C.M. and Dale, C.J., 1996, "Commercial Microelectronics Technologies for Applications in the Satellite Radiation Environment", Retrieved in January 27, 2015, from http://nepp.nasa.gov/DocUploads/0754D677-D2AF-4778B910A6CED2EA7458/apsen.pdf

Litwiller, D., 2009, "CCD vs. CMOS”, Photonics Spectra, Vol. 35, No. 1, pp. 154-158.

Marshall Space Flight Center, 2006, "Design Standard for Rigid Printed Circuit Boards and Assemblies", National Aeronautics and Space Administration (NASA), Pennsylvania, USA.

Montenbruck, O., Gill, E. and Kroes, R., 2005, "Rapid Orbit Determination of LEO Satellites Using IGS Clock and Ephemeris Products", GPS Solutions, Vol. 9, No. 3, pp. 226-235.

Pence, L.C., 2010, "Definition of the Flexible Image Transport System
(FITS), version 3.0", Astronomy \& Astrophysics, Vol. 524, pp. 1-40. doi: 10.1051/0004-6361/201015362

Petkov, M.P., 2003, "The Effects of Space Evironments on Electronic Components", Retrieved in Jan, 06, 2015, from http://trs-new.jpl. nasa.gov/dspace/bitstream/2014/7193/1/03-0863.pdf

Rønning, S.S., 2012, "Optimizing an Infrared Camera for Observing Atmospheric Gravity Waves from a CubeSat Platform", Ph.D. Thesis, Norwegian University of Science and Technology, Trondheim, Norway.

Saundau, R., 2008, "Status and Trends of Small Satellite Missions for Earth Observation", Acta Astronautica, Vol. 66, No. 1-2, pp. 1-12. doi:10.1016/j.actaastro.2009.06.008

Scholz, A., Leyb, W., Dachwaldb, B., Miaua, J. and Juangc, J., 2010, "Flight Results of the COMPASS-1 Picosatellite Mission", Acta Astronautica, Vol. 67, No. 9-10, pp. 1289-1298.

Schor, D., Kinsner, W. and Thoren, A., 2009, "Satellite Ground Station Emulator: an Architecture and Implementation Proposal", Proceedings of the Canadian Conference on Electrical and Computer Engineering, St. John's, Canada.

Selva, D. and Krejci, D., 2012, "A Survey and Assessment of the Capabilities of Cubesats for Earth Observation", Acta Astronautica, Vol. 74, pp. 50-68. doi:10.1016/j.actaastro.2011.12.014

Semiconductor Industry Association, 2014, International Technology Roadmap for Semiconductors (ITRS). Retrieved in January 27, 2015, from http://www.itrs.net/

Seo, H.S., Rhee, J., Han, E.S. and Kim, I.S., 2012, "Thermal Failure of the LM117 Regulator under Harsh Space Thermal Environments", Aerospace Science and Technology, Vol. 27, No. 1, pp. 49-56. doi:10.1016/j.ast.2012.06.006

She, M.M., 2003, "Semiconductor Flash Memory Scaling", University of California, Berkeley, USA.

Stras, L.N., Kekez, D.D., Wells, G.J., Jeans, T., Zee, R.E., Pranajaya, F.M. and Foisy, D.G, 2003, "The Design and Operation of the Canadian Advanced Nanospace eXperiment (CanX-1)", Proceedings of the AMSAT-NA 21st Space Symposium, Toronto, Canada.

Tennyson, R.C., 1995, "Composites in Space Challenges and Opportunities", Proceedings of the 10th International Conference on Composite Materials, Whistler, Canada.

Woellert, K., Ehrenfreund, P., Ricco, A.J. and Hetzfeld, H., 2011, "Cubesats: Cost-Effective Science and Technology Platforms for Emerging and Developing Nations", Advances in Space Research, Vol. 47, No. 4, pp. 663-684. doi:10.1016/j.asr.2010.10.009 